\title{
Morphology of the ultraviolet Io footprint emission and its control by Io's location
}

\author{
Jean-Claude Gérard, ${ }^{1}$ Adem Saglam, ${ }^{1}$ Denis Grodent, ${ }^{1}$ and John T. Clarke ${ }^{2}$ \\ Received 20 July 2005; revised 6 December 2005; accepted 11 January 2006; published 1 April 2006.
}

[1] A total of 74 images of the ultraviolet footprint of the Io flux tube (IFT) on Jupiter's upper atmosphere made with the Space Telescope Imaging Spectrograph on board the Hubble Space Telescope have been analyzed to characterize their location, morphology, and brightness distribution. The observations cover a wide range of central meridian Jovian longitudes and Io orbital positions and include north and south footprint emissions. Comparing the location of the IFT with that expected from the VIP4 model of the Jovian magnetic field, we find that the lead angle is generally not significantly different from zero in the System III longitude sector $125^{\circ}-195^{\circ}$. Instead, the lead angles reach about $8^{\circ}$ in the $50^{\circ}$ sector, coinciding with a region of possible magnetic anomaly. We observe that the brightness of the main footprint shows intrinsic intensity changes that appear to be controlled by the system III longitude of Io and its position above or below the center of the torus. The size of the primary spot magnetically maps into a region varying from 1 to over 10 Io diameters in Io's orbital plane. Multiple footprints are observed with varying brightness relative to the mean spot. The number of spots is found to increase as Io gets closer to the torus outer edge facing the spots. The separation between the first and second spots is typically $1^{\circ}-3^{\circ}$ of longitude and increases when Io is displaced from the torus center in the direction of the IFT signature. These features confirm that Alfvén waves play an important role and generate energization of precipitated electrons. However, the observed variation of the FUV spot structure with Io's position appears inconsistent with models where reflections of Alfvén wings occur between the torus boundary and Jupiter's ionosphere. Instead, the multiple spots apparently correspond to electron precipitation generated by Alfvén waves reflected inside the plasma torus.

Citation: Gérard, J.-C., A. Saglam, D. Grodent, and J. T. Clarke (2006), Morphology of the ultraviolet Io footprint emission and its control by Io's location, J. Geophys. Res., 111, A04202, doi:10.1029/2005JA011327.

\section{Introduction}

[2] The discovery of an infrared spot near the foot of the Io flux tube (IFT) [Connerney et al., 1993] opened a new window to remotely sense the interaction between Io, the Jovian magnetic field and Jupiter's upper atmosphere. The feature was observed to move across the Jovian disk as expected from the Io orbital motion. Bright far ultraviolet auroral emissions associated with the footprint were subsequently observed with the Hubble Space Telescope WFPC-2 [Clarke et al., 1996] and post-corrective optics space telescope axial replacement (COSTAR) faint object cameras [Prangé et al., 1998]. More recently weaker footprint FUV emissions, associated with Europa and Ganymede, have also

\footnotetext{
${ }^{1}$ Laboratoire de Physique, Atmosphérique et Planétaire, Université de Liège, Liège, Belgium.

${ }^{2}$ Center for Space Physics, Boston University, Boston, Massachusetts, USA.

Copyright 2006 by the American Geophysical Union. 0148-0227/06/2005JA011327\$09.00
}

been identified by Clarke et al. [2002]. The visual imager on board Galileo also detected the presence of emission at visible wavelengths [Vasavada et al., 1999]. Measurements of the infrared IFT footprint in the southern hemisphere [Connerney et al., 1993] indicated that the footprint leads the undisturbed field line connecting Io to the Jovian ionosphere by $15^{\circ}$ to $20^{\circ}$ in the direction of Io's orbital motion. These values were obtained from the comparison with the expected footprint location based on the O6 magnetic field model. However, estimates of the lead angle rely on the availability of a magnetic field model providing accurate values of the longitude of the undisturbed IFT footprint corresponding to the instantaneous position of Io on its orbit. The VIP4 model [Connerney et al., 1998] is based on a fit to Pioneer and Voyager magnetometer data and IFT footprint latitude measurements. When compared to the VIP4 model, the locus of the FUV footprints is closely coincident with the projections of the footprints mapped along the unperturbed Jovian magnetic field. A longitudinal lead angle (in the plasma flow direction) varying from the $0^{\circ}$ to $15^{\circ}$ in both the North and the South is observed from the instantaneous magnetic pro- 
jection, depending on the system III longitude of Io [Clarke et al., 1998]. However, some longitudinal sectors were poorly covered, in particular in the $260^{\circ}-360^{\circ}$ system III sector. The southern lead angle appeared largest when Io was located north of the torus, corresponding to a maximum column of plasma crossing between Io and its footprint. Inversely, the smallest northern lead angles were observed when Io was located north of the torus, although the available data points did not cover a wide range of longitudes.

[3] With the increased sensitivity of the Space Telescope Imaging Spectrograph (STIS) camera, images also show a long trailing tail extending downstream of the footprint along the magnetic footprint of Io's orbit over sometimes at least $100^{\circ}$ in longitude [Clarke et al., 2002]. This trailing emission is presumably the result of transfer of angular momentum to the plasma picked up from Io by the corotating magnetic field of Jupiter [Hill and Vasyliūnas, 2002; Delamere et al., 2003]. Previous observations of the FUV IFT footprint emission with the Wide Field Planetary Camera (WFPC2) have characterized it by a typical brightness of about $40-250$ kilorayleighs (kR), averaged over a $0.5 \times 0.5 \operatorname{arc~sec}^{2}$ area. The total electron power associated with the Io footprint emission varies from $6 \times 10^{9} \mathrm{~W}$ to $5 \times$ $10^{10} \mathrm{~W}$. No clear correlation between the footprint brightness and the sub-Io longitude could be identified by Clarke et al. [1998], as they were considerable time variations superimposed a possible longitudinal dependence. Analysis of the signature of hydrocarbon absorption in spatially resolved spectra of the IFT footprint [Dols et al., 2000; Gérard et al., 2002] suggests that the energy of the electrons causing the footprint emission is on the order of $50 \mathrm{keV}$, in any case less than the energy of auroral electrons exciting the main oval and the polar emission inside the oval.

[4] The interaction between Jupiter and Io has been extensively studied since the discovery that Jupiter's decametric emissions are controlled by the position of Io along its orbit. It was recently reviewed by Saur et al. [2004] and Kivelson et al. [2004] and will be only briefly summarized here. A current of $\sim 5 \times 10^{6} \mathrm{~A}$ was observed to flow between the two bodies by Voyager 1 [Acuña et al., 1981]. The interaction was initially explained by a unipolar inductor model [Goldreich and Lynden-Bell, 1969] where the motion of Io across the Jovian magnetic field lines generates a $\sim 500 \mathrm{keV}$ electric potential driving currents along Jupiter's field lines and a DC current circuit. The current circuit is closed in Jupiter's ionosphere and flows back to Io. The lead angle of the footprint (in the corotation direction) was initially calculated to exceed $12^{\circ}$, depending on the conductivities of the ionospheres of Io and Jupiter, but not on the density of the plasma torus. Another model involves the generation of Alfvénic wave propagating from Io to Jupiter [Neubauer, 1980; Belcher et al., 1981]. The finite propagation time along magnetic field lines partly depends on the density of the torus plasma encountered by the wave along its path. Saur [2004] showed that both models are consistent with the low flow speed of the flux tubes in Io's wake measured by Galileo [Frank et al., 1996].

[5] The importance of the two processes (steady current loop and Alfvén wings) depends on the relative values of the wave transit time to the Jovian ionosphere and the time for the plasma to flow across Io. A hybrid model by Crary and Bagenal [1997] suggested that the interaction begins as an Alfvén disturbance near Io, evolving into a steady current loop downstream. In situ measurements indicate that the interaction is additionally controlled by mass loading in the vicinity of Io [Goertz, 1980; Saur et al., 1999; Russell and Huddleston, 2000], which drives strong parallel currents along the flux tubes of the associated Alfvénic disturbance [Neubauer, 1980; Belcher et al., 1981]. Strongly field-aligned energetic electron beams toward and from Jupiter, possibly related to the footprint emission, were detected in the wake of the plasma flows past Io with Galileo [Williams et al., 1996; Frank and Paterson, 1999]. The power carried by the low-energy electron beams was estimated on the order of $3 \times 10^{10} \mathrm{~W}$. Crary [1997] proposed a model where the source of the infrared and FUV Io footprint is a precipitated electron beam accelerated by the wave's parallel electric field through a process of repeated Fermi accelerations. $\mathrm{He}$ predicted a total electron power precipitation of $\sim 10^{11} \mathrm{~W}$ with an average energy of about $80 \mathrm{keV}$. Chust et al. [2005] recently suggested that the presence of high-frequency/ small-scale waves observed by Galileo is a signature of filamentation of the Alfvén wings before they are reflected off the sharp boundary gradient of the Io torus. The resulting high-frequency/small-scale electromagnetic waves are able to accelerate electrons once they reach Jovian high latitudes.

[6] Multiple footprints, presumably resulting from Alfvén wave reflections between the torus and Jupiter's ionosphere, have been observed both in the infrared [Connerney and Satoh, 2000] and the ultraviolet. STIS images occasionally showing a complex, sometimes doubly peaked, structure of the auroral Io footprint emissions have been presented by Clarke et al. [2002]. However, the occurrence and characteristics of these multiple spots, which provide potentially important constraints on models of the Io-Jupiter interaction, have not been documented so far. They have generally been interpreted as the signature of Alfvén waves reflected between the northern and southern edges of a Jovian field line or between Jupiter's ionosphere and the facing boundary of the plasma torus. In this study, we use STIS observations covering a wide range of central meridian Jovian longitudes and Io orbital positions to characterize the location, morphology and brightness distribution of the IFT footprints. In particular, we analyze the lead/lag angle of the footprint, the presence of multiple spots and the variation of their angular separation.

\section{Observations}

[7] Far ultraviolet images of the high-latitude Jovian regions collected with the Space Telescope Imaging Spectrograph from December 2000 to February 2003 have been analyzed. The images were collected with the FUV MultiAnode Microchannel Array (MAMA) photon counting detector providing a $1024 \times 1024$ pixels image. The point spread function of the camera is about 3 pixels wide and each pixel subtends an angle of 0.024 arcsec. Therefore the spatial resolution ranges from $\sim 0.2^{\circ}$ of system III longitude at $60^{\circ} \mathrm{N}$ on the central meridian to $\sim 0.8^{\circ}$ of longitude, $75^{\circ}$ 


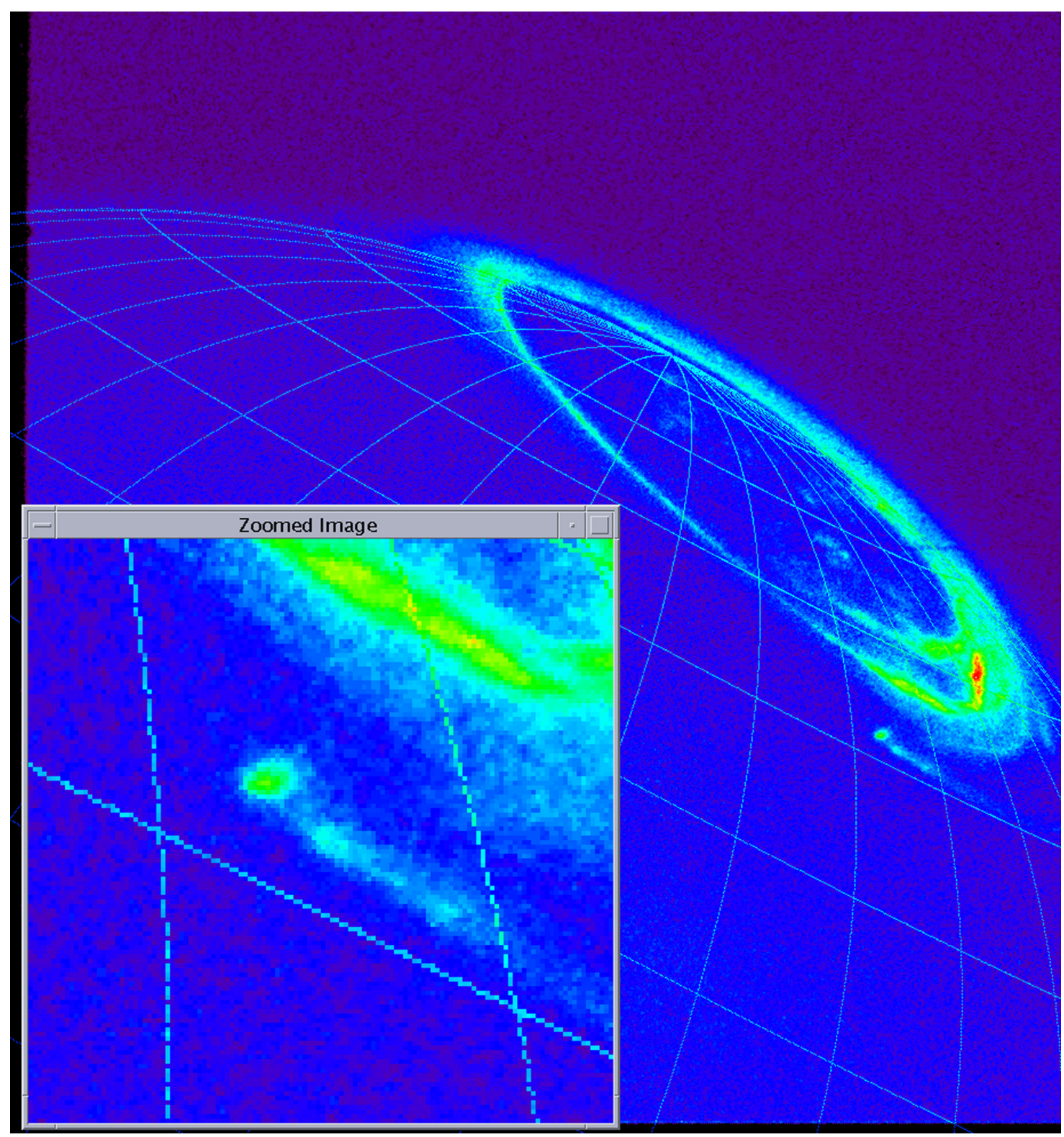

Figure 1. Jovian ultraviolet auroral emission observed in the north polar region on 12 December 2000 at 1205 UT with the Hubble Space Telescope-Space Telescope Imaging Spectrograph camera. The exposure time was $110 \mathrm{~s}$, and the planetary central meridian longitude was $209^{\circ}$ with Io $\lambda_{\text {III }}=184^{\circ}$ and a latitude of $6.2^{\circ} \mathrm{N}$ of the torus center. The Io footprint and trailing tails are clearly observed equatorward of the main auroral oval and the diffuse aurora. (insert) Zoom on the footprint structure showing three additional spots located to the right (in the corotation direction) of the primary spot. The third spot is weaker than the second and fourth ones.

from the central meridian. The instrument was operated either in the ACCUM mode where photons counts are integrated in each pixel or in the TIMETAG mode, which provides an event stream with fast time resolution. The exposures were obtained using the $\mathrm{SrF}_{2}$ filter rejecting the HI Lyman- $\alpha$ emission or in the MAMA clear mode transmitting light from 115 to $180 \mathrm{~nm}$. The sensitivity threshold is on the order of $1 \mathrm{kR}$. A total of 94 exposures in the northern hemisphere and 81 in the southern hemisphere were collected during the period considered. All of the 175 images showed a FUV Io footprint when the geometry was appropriate. However, only 58 in the north and 16 in the south showed a footprint signature located at least $10^{\circ}$ from the limb. Locations closer to the limb cause increasing inaccuracy on the spot's location and intense limb brightening requiring extensive but uncertain corrections. Processing of STIS images and the background subtraction were described by Clarke et al. [2002] and Grodent et al. [2003]. The count rate was converted into physical units of brightness (kR), energy flux ( $\mathrm{mW} \mathrm{m}^{-2}$ ), or total power (W) on the basis of the instrumental response, in-flight calibrations, and laboratory $\mathrm{H}_{2}$ spectra.

[8] We assume that the Io footprint is excited by electrons accelerated along the magnetic field lines connecting Io and 


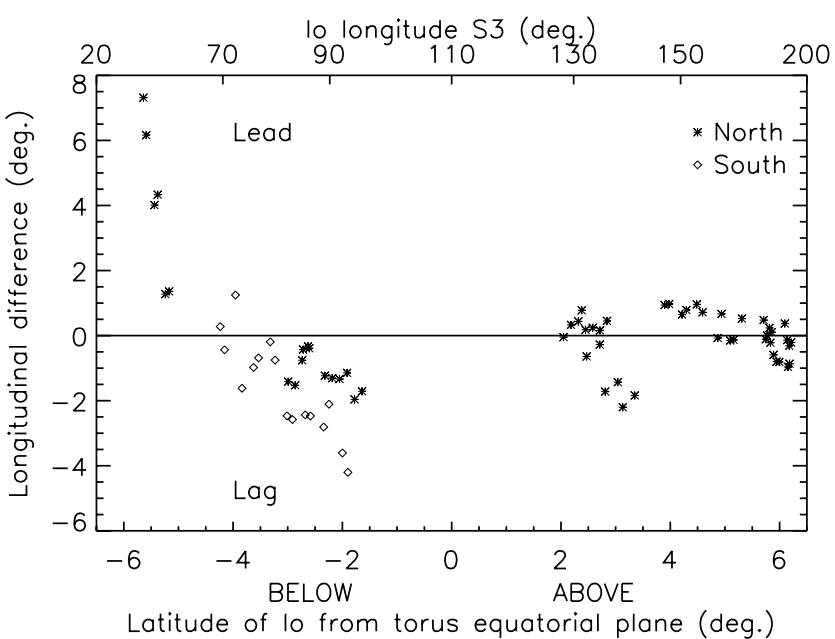

Figure 2. Difference in longitude between the observed Io footprint emission and the Io mapping from the VIP4 magnetic field model. Positive values correspond to lead angles, where the observed main spot is located forward in the corotation direction compared to the unperturbed magnetic field. Stars correspond to north emission, and diamonds correspond to south.

the Jovian ionosphere. Spectral measurements made with STIS have shown that the ultraviolet photons emitted in the footprints are only weakly absorbed by overlying hydrocarbons [Gérard et al., 2002]. In this case, earlier studies [Gérard and Singh, 1982; Waite et al., 1983; Grodent et al., 2001] indicate that the efficiency of the electron energy conversion into $\mathrm{H}_{2}$ Lyman and Werner band excitation is close to $10 \mathrm{kR} /$ incident $\mathrm{mW} \mathrm{m}^{-2}$ for incident electron energies between 10 and $100 \mathrm{keV}$, decreasing at lower electron energies.

[9] Figure 1 shows an example of the auroral emission observed in the north on 14 December 2000. At the time of the $110 \mathrm{~s}$ exposure, Io was located at a system III longitude of $209^{\circ}, 6.1^{\circ}$ south of the torus center. The Io footprint and the trailing tail are clearly seen eastward of the central meridian and equatorward of the auroral emissions. We note the progressive decrease of the downstream tail brightness. The inserted zoomed image provides a detailed view of the region of the IFT footprint. The main peak associated with the primary spot reaches $155 \mathrm{kR}$ (uncorrected for view angle) and dominates the IFT emission. Three weaker spots stand up above the tail emission $1.8^{\circ}, 3.8^{\circ}$ and $5.3^{\circ}$ of longitude downstream of the main spot. Remarkably, the third spot $\left(3.8^{\circ}\right.$ downstream) is weaker than the other two neighbor spots. The morphology of multiple footprints in the FUV IFT emission is discussed in section 4.

\section{Lead/Lag Angle}

[10] As mentioned before, the value of the lead angle of the footprint from the mapped location of the undisturbed magnetic footprint may provide constraints on the nature of the Io-Jupiter interaction. Lead angles less than $12^{\circ}$ are predicted by models where currents are carried by Alfvén wings, with a finite propagation time between Io and Jupiter's ionosphere [Goertz, 1980; Neubauer, 1980;
Belcher et al., 1981]. Instead, models where the currents are instantaneously set up between the two bodies predict lead angles on the order of $15^{\circ}$ [Goldreich and Lynden-Bell, 1969]. Lags are not predicted by models of Io-Jupiter magnetic field interaction. Figure 2 shows the lead/lag angle calculated by comparing the SIII longitude of the first spot of the FUV IFT footprint with the location of the foot point of the field line passing through Io predicted by the VIP4 model [Connerney et al., 1998]. The 2000-2001 data and the 2003 observation are represented by different symbols to account for possible brightness variations induced by changes in volcanic activity modifying the neutral particle production rate and thus the torus plasma density.

[11] The accuracy of the SIII longitude of the footprint is estimated to range from $\sim 0.5^{\circ}$ near the central meridian to $2.5^{\circ}, 15$ degrees from the limb. Globally, $84 \%$ of the data points range between $+2^{\circ}$ and $-2^{\circ}$. Taking into account the additional $2^{\circ}-3^{\circ}$ uncertainty of the VIP4 model, most observations do not show significant departure from a zero lead angle in the System III longitude sector $125^{\circ}-195^{\circ}$ and are thus compatible with the Alfvén wing model. A set of 8 south observations where the IFT lags behind the expected location of the undisturbed field line is observed in the $\lambda_{\text {III }} 82^{\circ}-93^{\circ}$ sector. It is possible that somewhat larger uncertainties associated with the VIP4 model in this sector combined with our determination of the footprint longitude, explain these larger lag angle values. Probably more significant are the larger lead angles (up to $7.4^{\circ}$ ) observed for the south footprint in the $48^{\circ}-56^{\circ}$ SIII sector, corresponding to an Io latitude of more than $5^{\circ}$ below the torus center. These lead angles appear to exceed the combined uncertainties of the lead angle determination. This uncertainty in the $\lambda_{\text {III }}$ of this group of footprints is on the order of $1^{\circ}$. The particular geometry of these observations

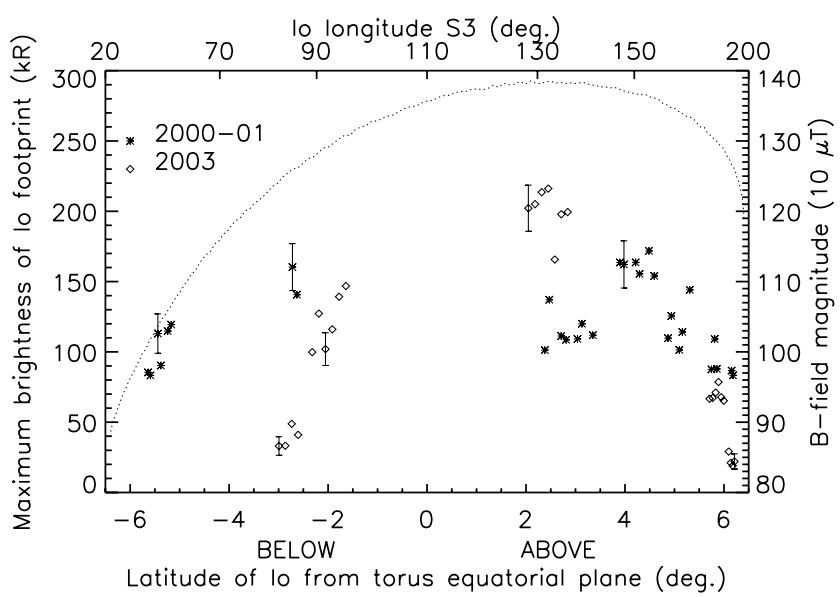

Figure 3. Maximum brightness of the Io footprint versus system III Io longitude and latitude from the torus plane for two time periods. The stars correspond to observations made from January 2000 to December 2001, and the diamonds correspond to data collected in February 2003. The spot brightness has been corrected assuming a cosine dependence on the view angle. The error bars correspond to a 1-sigma statistical uncertainty in the number of counts. The dotted line shows the magnetic field intensity at the footprint location derived from the VIP4 model. 


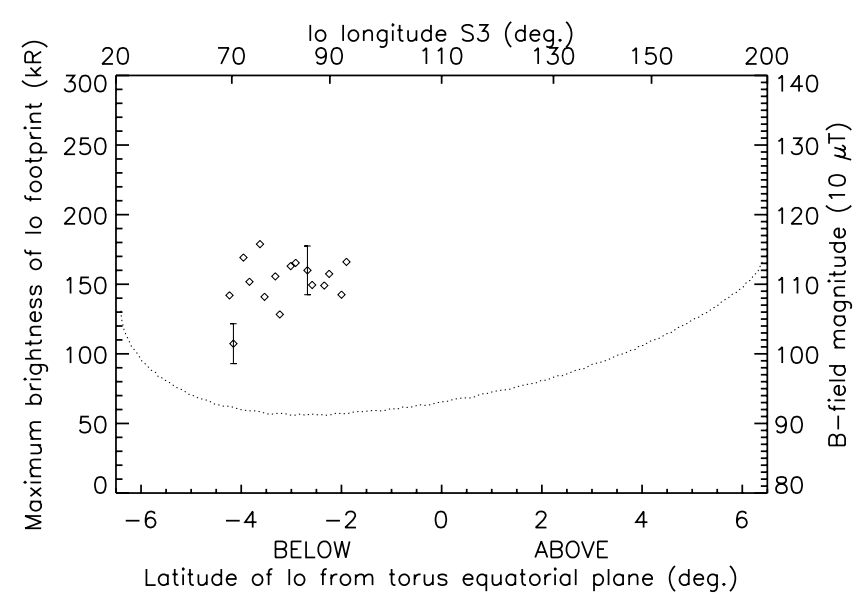

Figure 4. Same as Figure 3 for the south footprint emission.

maximizes the path of the Alfvén wing in the torus plasma, presumably leading to maximum propagation time of the Alfvén disturbance. However, we note that this longitudinal sector also coincides with the region of a possible magnetic field anomaly, unaccounted for by the VIP4 model, deduced from the "kink" observed in the shape of the north auroral main oval [Grodent et al., 2003].

\section{Brightness and Multiple Spots}

[12] We now examine the distribution of the FUV brightness along the trailing tail emission associated with the IFT footprint and its possible dependence on Io's position. Figure 3 shows the brightness of the north main footprints. Measurements made from December 2000 to January 2001 and in February 2003 are represented by different symbols, as part of the scatter may be caused by long-term variations of the plasma characteristics in the torus. A correction by the cosine of the emission angle has been applied to account for the slant observing geometry. The corrected values of the brightness are shown in Figure 3 and range from 23 to $230 \mathrm{kR}$ of $\mathrm{H}_{2} B$ and $C$ state emission. The corresponding precipitated electron energy fluxes are in the range 2.3$23 \mathrm{~mW} \mathrm{~m}^{-2}$. The lowest values were measured in $2000-$ 2001 in the $180^{\circ}-190^{\circ} \lambda_{\text {III }}$ sector (Io latitude close to $6^{\circ} \mathrm{N}$ ). The highest-brightness points in our sample correspond to measurements collected in 2003 near $\lambda_{\text {III }}=130^{\circ}$ (Io latitude is $\left.2.0^{\circ}-2.5^{\circ} \mathrm{N}\right)$. A large scatter of values is observed near Io latitudes of $3^{\circ}-2^{\circ} \mathrm{S}$ and $2^{\circ}-2.5^{\circ} \mathrm{N}$. The intensities associated with measurements made in 2000 near $\lambda_{\text {III }}=130^{\circ}$ are however subject to larger uncertainties since the IFT spots were located as close as $15^{\circ}$ from the limb. In spite of the temporal variations and scatter, it can be noted that in both (2000-2001 and 2003) subdata sets, the low-brightness data points are associated with Io's positions most distant from the torus center. No significant correlation was found between the peak brightness of the footprint and its lead angle (as determined from the VIP4 field model). Figure 4 shows the cosine corrected intensities for the south IFT footprint. All points belong to the $\lambda_{\text {III }} 69^{\circ}-93^{\circ}$ Io longitude sector. The total power carried by electrons precipitated in the primary footprint spot varies from 0.4 to $8 \mathrm{GW}$. Unlike the infrared footprint, no systematic FUV brightness difference is observed between the north and south footprint brightness, although the size of the data set is too small to definitely conclude. If confirmed, this lack of hemispheric asymmetry contradicts the idea that the Io interaction is modulated by the surface field strength and the corresponding change of the equatorial loss cone aperture. In this case, the south spot would be expected to be weaker when the north emission is bright and vice versa. Similarly, the north spot does not show a brightness decrease where the $B$ field intensity is higher in the $70^{\circ}-100^{\circ} \lambda_{\text {III }}$ sector. For comparison the $B$ field intensity at the footprint from the VIP4 model has been plotted as a dotted line in Figures 3 and 4 . If any dependence on $B$ is suggested in Figure 3, the spot brightness correlates rather than anticorrelates with the intensity of $B$. In the absence of the expected longitudinal and hemispheric dependence, we conclude that the strength of the magnetic field in the ionosphere does not appear to play a key role in the control of the FUV emission brightness.

[13] Earlier studies [Connerney and Satoh, 2000; Clarke et al., 2002] have indicated the occasional presence of multiple infrared and FUV spots located downstream of the first IFT footprint. With the high sensitivity of the STIS camera, it is now possible to get a global picture of the morphology of the footprint ultraviolet emission. Following attempts to correlate the footprint morphology to various orbital and observational parameters, we find that the location of Io in its torus is the dominating factor. Figure 5 illustrates the evolution of the morphology of the emissions associated with the IFT in both hemispheres as a function of the position of Io in the torus and shows a sample of 16 north and 16 south images out of 74 , but it clearly illustrates the global behavior observed over the entire data set. We first examine the evolution of the footprint structure in the northern hemisphere for Io magnetic latitudes ranging from $6.1^{\circ} \mathrm{N}$ to $2.2^{\circ} \mathrm{S}$. A clear trend is readily observed: when Io is located below the torus plane, a single elliptic spot is observed with its longer extension along the tail. As Io moves above the center of the torus, a secondary peak appears near $2.5^{\circ} \mathrm{N}$. Note that the false color scale is identical for all brightness traces and does not always show the double spot structure present in the most downstream emission. Progressively, one and ultimately three or more secondary spots are observed with increasing Io distance from the torus plane. It is possible that this series of weak spots correspond to the short, faint tail observed with the Galileo visible imager when Jupiter's dipole was tilted toward Io [Vasavada et al., 1999]. The evolution of the south spot structure is also illustrated in Figure 5. In this case, all available observations were made when Io was positioned south of the torus, at latitudes ranging from $4.2^{\circ}$ to $1.9^{\circ} \mathrm{S}$. The same trend as for the north footprint is observed. As Io moves farther south from the torus plane, the initially main double-headed spot becomes more elongated, splitting into two individual structures at Io latitudes exceeding about $2.5^{\circ}$. The brightness of the secondary spots in the south is relatively higher than in the north as can be seen by comparing Figure 5 (left) (north) and Figure 5 (right) (south). Examining the morphology of the main and first secondary spots closer, it is also apparent that, in the south, the centers of the two spots are generally not coal- 


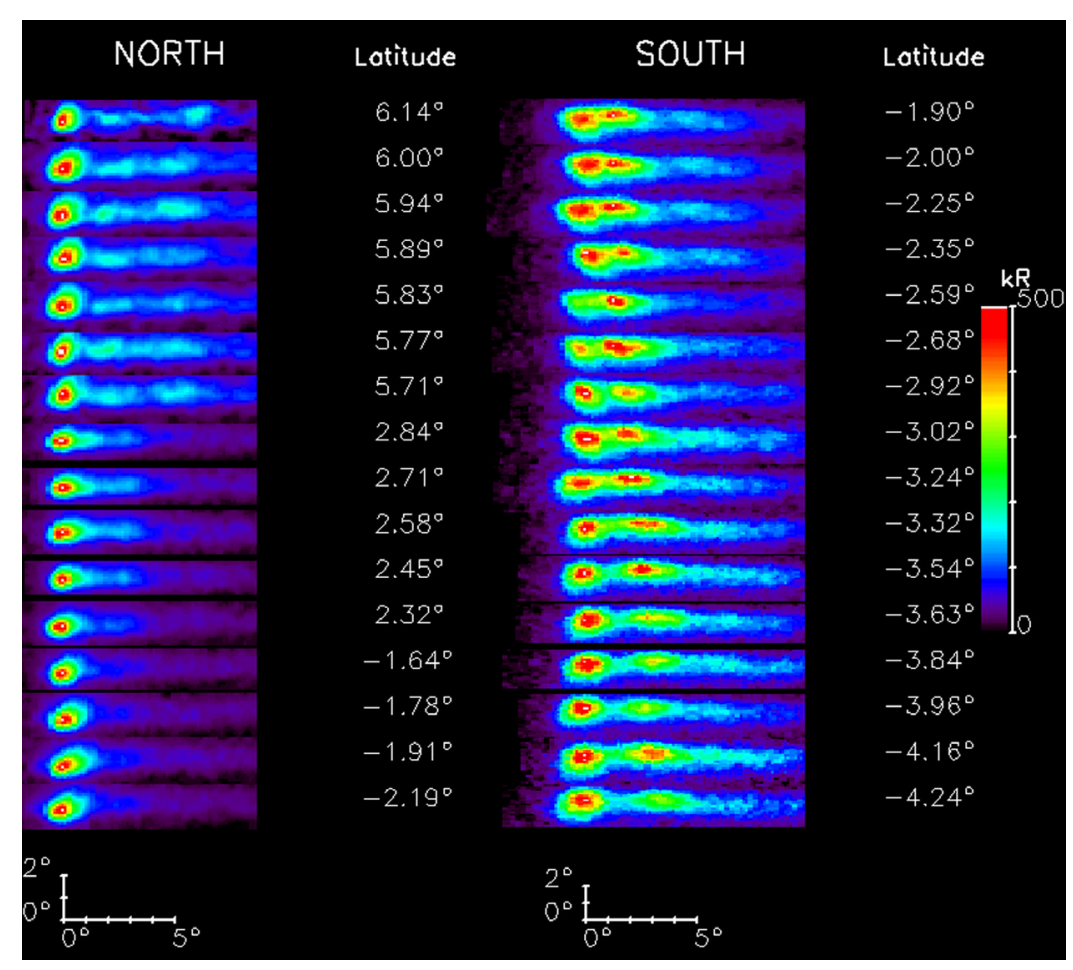

Figure 5. Samples of rectified traces of Io footprint emissions ordered by values of the Io distance from the torus plane. (left) North footprint and (right) south footprint. The data points for the north footprints were collected in February 2003; those for the south were obtained in December 2000 and January 2001. The scales in degrees of latitude and longitude are indicated at the bottom. The color scale refers to the uncorrected brightness.

igned with the orientation of the downstream tail. This feature is readily observed on most of the example brightness traces shown in Figure 5. It is also noticeable that the secondary spots in the south are generally more elongated (along the Io orbital motion direction) than the primary spot, for all longitudes of Io. The same characteristic is observed, although less conspicuous, in the weaker north secondary spots.

[14] The brightness distribution of the north IFT footprint and trailing tail observed on 28 December 2000 at 1230 UT is shown in Figure 6. At this time, Io was located at $\lambda_{\text {III }}=174^{\circ}, 5.8^{\circ}$ above the torus plane. Two secondary peaks are separated by $1.7^{\circ}$ and $4.1^{\circ}$ of longitude from the main spot in the corotation direction. Both are about $35 \%$ as bright as the main peak. Additional peaks are possibly present further downstream but are less easily distinguished from the background tail emission. The dependence of the number of spots on Io's magnetic latitude is shown in Figure 7 for both the north and the south footprints. Although some ranges of Io distances from the torus plane are not covered, the trend illustrated in Figure 5 is clearly apparent. A single spot is detected in the north for locations of Io south of the torus center For northern Io latitudes, the number of spots gradually increases from 2 to 4 as Io moves away from the centrifugal equator. Symmetrically, in the south, at least three spots are observed for southern Io latitude. The global picture is thus that the number of IFT footprints increases when Io approaches the torus boundary on the side facing the footprint in Jupiter's ionosphere.
[15] We now examine the variation of the distance between multiple spots. The longitudinal distance between the first and the second footprints in both hemispheres is presented in Figure 8. It confirms and quantifies the picture

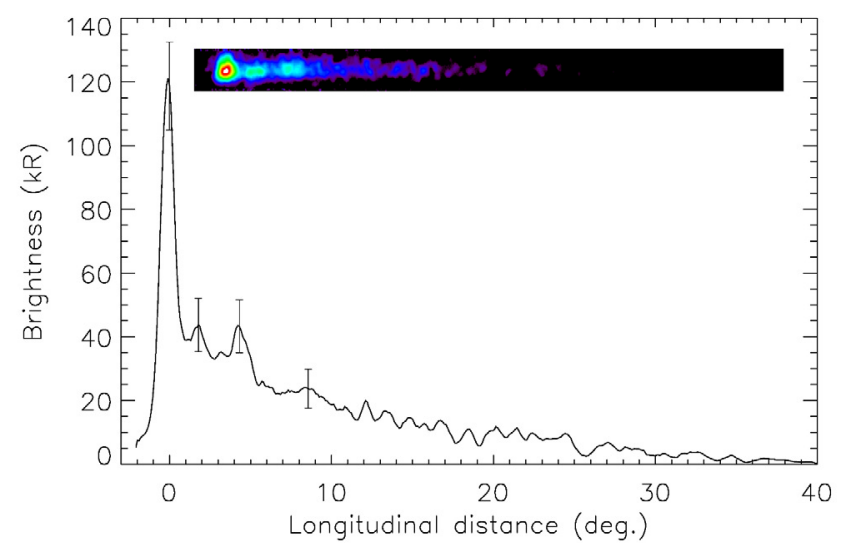

Figure 6. Brightness distribution of the north Io flux tube (IFT) footprint and trailing tail observed on 28 December 2000 at 1230 UT. Io was located at $\lambda_{\mathrm{III}}=174^{\circ}, 5.8^{\circ}$ north of the torus plane. The curve has been smoothed on 3 pixels. The error bars correspond to a 1-sigma statistical uncertainty in the number of counts. Two secondary peaks are observed $1.7^{\circ}$ and $4.1^{\circ}$ downstream of the primary spot. Insert shows the two-dimensional brightness distribution in the raw image in the vicinity of Io's footprint and trailing tail. 


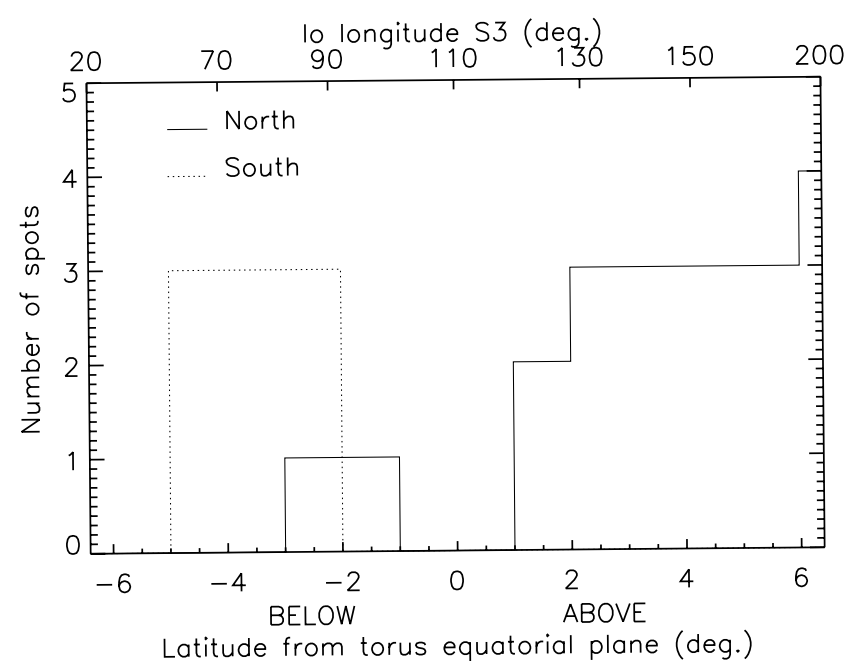

Figure 7. Histogram of the number of spots observed in the north and south IFT footprint emission.

described in Figure 5. The interspot distance steadily increases with Io's latitude. Minimum values on the order of $0.9^{\circ}$ are observed in the north while maximum distances are as large as $2.2^{\circ}$. No data point exists for southern Io latitudes as a single spot is seen in the case (Figure 5). A linear regression line is plotted with a correlation coefficient $r=0.90$, which is statistically significant at the $95 \%$ confidence level. Similarly, the southern spot interspot distance increases for increasing southern Io distance from the torus plane. A linear correlation coefficient $r=0.96$ is obtained in this case. The distance of the third spot, when present, is more difficult to determine. In the south, the separation between the third and second spots is generally comparable to that between the first and the second one. In the north, the second spot tends to be closer to the first than to the third one. A more detailed discussion of this aspect is left to a more systematic study.

[16] Summarizing our findings, the IFT footprint shows a structure that depends on the location of Io. The number of observed UV footprints and the interspot distance are clearly correlated with Io's orbital position. This variation is interpreted in terms of the distance of Io from the torus plane. The measured distance separating the spots is typically between $1^{\circ}$ and $3^{\circ}$ of Jovian longitude, corresponding to a difference of Alfvén wing travel time along the magnetic field line of about $130-400$ s from Io to Jupiter's ionosphere.

\section{Spatial Extent of the Footprint}

[17] The high spatial resolution and sensitivity of the STIS camera makes it possible to determine the spatial extent of the Io FUV footprint. The short exposure times required to image the footprint with the sensitive STIS camera is such that during typical $110 \mathrm{~s}$ exposures, Io moves along its orbit by only $0.3^{\circ}$, which maps as a footprint displacement between $0.13^{\circ}$ and $0.96^{\circ}$ of longitude in the north, depending on Io's SIII longitude. Therefore it is possible to estimate the intrinsic size of the Io interaction region causing electron acceleration and precip- itation. For this purpose, we assume that the magnetic field lines connecting Io to its footprint are not significantly disturbed so that the VIP4 model may be used. As an example, the primary spot illustrated in Figure 1 has a longitudinal full width at half maximum (FWHM) of $\sim 0.9^{\circ}$ and a width above the background emission of $\sim 1.3^{\circ}$, as determined from the spot brightness distribution along the direction of Io's trailing tail. In comparison, during the $110 \mathrm{~s}$ exposure, Io's motion in Jupiter's undisturbed magnetic field generates a $0.73^{\circ}$ displacement of its footprint. Consequently, the spot elongation maps into a length on the order of 1 Io diameter, indicating that the interaction region producing the primary spot is confined to the immediate vicinity of Io.

[18] Figure 5 shows that as Io moves equatorward of the northern torus edge, the primary (and ultimately only) north spot becomes more elongated in the tail direction, progressively mixing with the trailing tail and decreasing continuously. For example, on 16 December 2000 at 1100 UT, Io was $5.6^{\circ}$ south of the torus center and the FWHM size of the single footprint was as large as $8^{\circ}$ of longitude, mapping to about 12 Io diameters. In this case, however, it is not clear that the half width of the intensity distribution has the same physical meaning as for multiple detached spots. The nature of the interaction may be different with a predominance of the Alfvén wings and their reflected components when the plasma content between Io and the footprint is smaller, leading to a single spot and more continuous decrease of the precipitated energy with distance for the footprint when the wave path increases. We conclude that when the primary spot is clearly detached from the trailing tail, the region of interaction is generally limited to a few Io diameters. Hinson et al. [1998] determined from Galileo radio occultation measurements that the plasma is reaccelerated, reaching $95 \%$ of corotation speed over after distance of about 3 Io diameters downstream of Io. We thus note that this distance is comparable to or smaller than the size of the

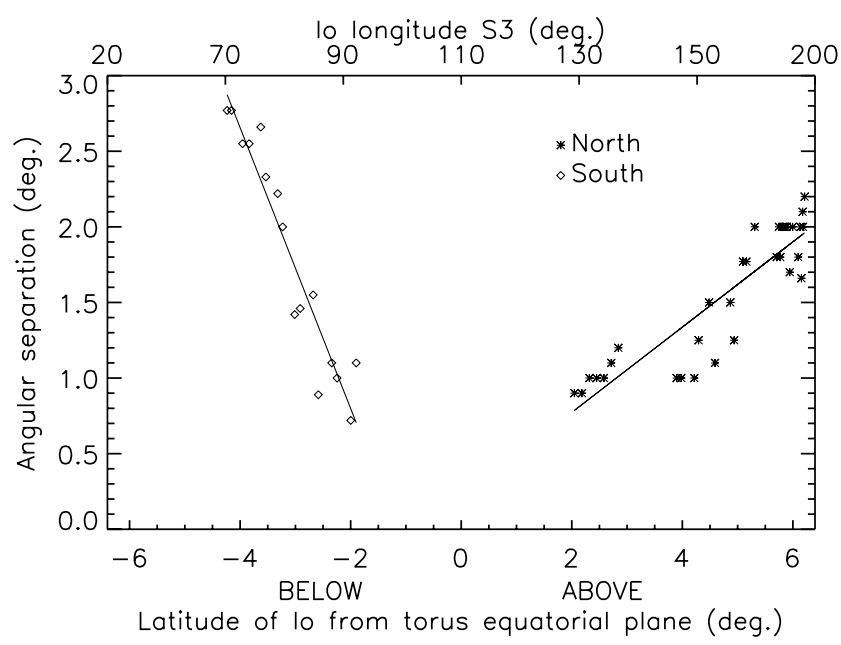

Figure 8. Angular distance between the first and second peaks of IFT emission in the north and in the south. The solid lines show the best linear fits to the data points. The correlation coefficients are 0.90 (south) and 0.96 (north). 


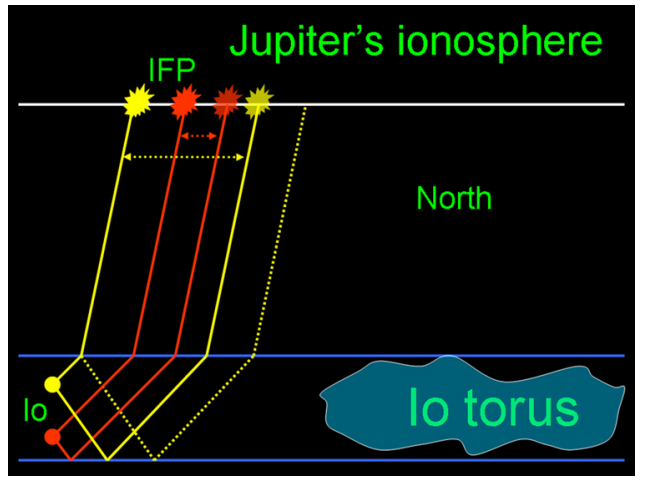

Figure 9. Schematic view of the Alfvén wave reflection pattern inside the high-density Io torus and energy propagation to Jupiter. When Io is positioned north of the torus center (in red), the separation between the first and second FUV spots is larger than when Io is located south of the center (in yellow). Distances are not to scale, and reflection angles are arbitrary.

"stagnation" region where the plasma in the wake of Io is progressively accelerated to corotation speed.

\section{Discussion}

[19] Comparing the location of the Io flux tube with that expected from the VIP4 model of the Jovian magnetic field, we find that the lead angle is generally small in the longitude sector $125^{\circ}-195^{\circ}$, in agreement with that expected for Alfvén wing propagation between Io and Jupiter's ionosphere. A larger angle is observed in the $\lambda_{\text {III }}$ sector near $50^{\circ}$ where the north lead angle reaches nearly $8^{\circ}$. This sector coincides with an apparent magnetic anomaly of the Jovian magnetic field. The dependence of the footprint brightness on the SIII longitude of Io leads us to suggest that the strength of the magnetic field in the ionosphere is of minor importance for the brightness of the FUV emission. Instead, the largest emitted power is associated with a location of Io close to the torus, where the interaction with the plasma is the strongest.

[20] The presence of multiple IFT footprints was also observed in the infrared [Connerney and Satoh, 2000] where as many as five secondary peaks were identified along Io's tail emission. The spots were equally separated by about $4^{\circ}-5^{\circ}$. It is not clear however whether this large separation is typical in the infrared footprint signature or is an unusual feature. The lower spatial resolution of the ground-based IR images may also preclude observation of smaller interspot separations such as those observed with Hubble Space Telescope (HST) in the ultraviolet. Multiple reflections of Alfvén waves have also been observed as arc structures in decametric emission observed from the ground [Leblanc, 1981; Queinnec and Zarka, 1998] and Voyager [Gurnett and Goertz, 1981; Bagenal and Leblanc, 1988]. These radio fringes were explained in terms of multiple reflections of the magnetic perturbation caused by Io's motion. In this view, radio emissions are modulated by Alfvén waves trapped between the upper outer edge of the torus and the Jovian ionosphere. The north fringes observed from the ground were distant by approximately $2 \mathrm{~min}$, corresponding to a longitudinal separation of $\sim 1^{\circ}$ of Jovian longitude.

[21] The distance between the first and the secondary spots is typically between $1^{\circ}$ and $3^{\circ}$ (Figures 4 and 6). As was shown in Figure 4, this distance appears to be controlled by the location of Io in the torus, both in the north and in the south. In the north, the main spot exhibits changes of brightness by about 1 order of magnitude. The brightness of the main spot does not anticorrelate with the intensity of the $B$ field at the IFT footprint. Instead, it appears controlled by the position of Io in the torus. $\mathrm{H}_{3}^{+}$ infrared measurements [Connerney et al., 1993] showed no measurable footprint in the north at longitudes where $B$ is significantly stronger than in the conjugate region in the southern hemisphere. It was speculated that a weaker $B$ field implies a larger loss cone and possibly enhanced electron precipitation. Our results suggest that the precipitation from the flux tube in the north is essentially dependent on the strength of the coupling with the torus plasma and therefore on the position of Io in the torus. The mapped size of the primary (or single) FUV footprint is on the order of a few Io diameters. This result is in agreement with the measured extent of about 3 Io diameters of the wake region where plasma is progressively brought to quasi-corotation.

[22] The longitudinal separation between the spots $\left(0.7^{\circ}-\right.$ $2.8^{\circ}$ ) provides constraints on where the Alfvén waves are reflected. Crary and Bagenal [1997] estimated the roundtrip Alfvén propagation time between Io and the Jovian ionosphere to $\sim 7.5,20$, or $33 \mathrm{~min}$ when Io is above, at the center, or below the torus center respectively. They suggest that, following the initial $1200 \mathrm{~s}$, a DC current is established which powers the majority of the DAM and auroral tail extending downstream. According to Gurnett and Goertz [1981], for an Earth-based observer the radio arcs would be separated by $6^{\circ}$ of Jovian longitude (40 min of Jovian rotation for a radio DAM observation), if reflections of Alfvén waves only take place between the northern and southern hemispheres. However, using $\mathrm{MA}=0.3$, a more appropriate value of the Alfvén Mach number, a distance of $12^{\circ}$ is calculated. Both values are larger than the angular separation reported here. Instead, Crary and Bagenal [1997] suggested that the waves are trapped between the ionosphere and the outer edge of the torus, leading to a time delay of 0.3 to $1.5 \mathrm{~min}$ corresponding to $0.14^{\circ}$ to $0.7^{\circ}$ of longitudinal separation. The distance between the FUV spots reported in this study lies between these two model values.

[23] Our observation that the FUV spot separation increases as Io moves away from the torus center toward the torus edge facing the footprint is also at variance with that expected from the shorter travel time of the disturbance at the Alfvén speed. This suggests that the reflections occur inside the torus in a density gradient region where the Alfvén speed rapidly increases and not between the torus boundary and Jupiter's ionosphere. If reflections take place inside the torus, the path and the travel time of the reflected wing increase as Io moves closer to the footprint, causing a larger separation between the spots (Figure 9). In this view, the decreasing brightness of the primary IFT spot for Io locations away from the torus plane suggests that the interaction between Io and the torus plasma maximizes near the torus center where the density is highest. We also note 
that the variation of the reflected component (multiple spots) may be related to the trend observed in the decametric radiation by Leblanc et al. [1994]. They observed that the south component tends to predominate when Io is near the center or south of the torus plane, while the north contribution is enhanced when Io is near the north edge.

[24] As mentioned before, Chust et al. [2005] suggested that Io's Alfvén wings are filamented prior to the first reflection by the sharp torus boundary, leading a continuous energy transfer from the large-scale Alfvén current system to high-frequency/small-scale electromagnetic waves before significant electron acceleration process takes place. These high-frequency electromagnetic waves are less reflected by the torus boundary and reach the high-latitude magnetosphere where they accelerate electrons through an unspecified process. This view is compatible with the observations reported in this study and our interpretation. It explains how significant power escapes from the torus in spite of the presence of the reflecting boundary at the outer edge of the torus.

[25] Acknowledgments. This work is based on observations with the NASA/ESA Hubble Space Telescope, obtained at the Space Telescope Science Institute (STScI), which is operated by the AURA, Inc. for NASA J.-C.G. and D.G. acknowledge support from the Belgian Fund for Scientific Research (FNRS), and J.T.C. acknowledges support from NASA STScl grant HST-GO-100140.01-A to Boston University. Funding for this research was provided by the PRODEX program of the European Space Agency. The STIS data used in this study were available from STScl archives (programs 8657, J.T. Clarke, P.I., and 9685, R.F. Elsner, P.I.). The authors thank B. Bonfond for discussions and both reviewers for their useful comments.

[26] Arthur Richmond thanks Joachim Saur and Ashwin Vasavada for their assistance in evaluating this paper

\section{References}

Acuña, M. H., F. M. Neubauer, and N. F. Ness (1981), Standing Alfvén wave current system at Io: Voyager 1 observations, J. Geophys. Res., 86, 8513.

Bagenal, F., and Y. Leblanc (1988), Io’s Alfvén wave pattern and the Jovian decametric arcs, Astron. Astrophys., 191, 311.

Belcher, J. W., et al. (1981), Plasma observations of the Alfvén wave generated by Io, J. Geophys. Res., 86, 8508.

Chust, T., A. Roux, W. S. Kurth, D. A. Gurnett, M. G. Kivelson, and K. K. Kurana (2005), Are Io's Alfvén wings filamented? Galileo observation, Planet. Space Sci., 53, 395.

Clarke, J. T., et al. (1996), Far-ultraviolet imaging of Jupiter's aurora and the Io "footprint", Science, 274, 404.

Clarke, J. T., et al. (1998), Hubble Space Telescope imaging of Jupiter's UV aurora during the Galileo orbiter mission, J. Geophys. Res., 103, 20,217.

Clarke, J. T., et al. (2002), Ultraviolet emissions from the magnetic footprints of Io, Ganymede, and Europa on Jupiter, Nature, 415, 997.

Connerney, J. E. P., and T. Satoh (2000), The $\mathrm{H}_{3}^{+}$ion: Remote diagnostic of the Jovian magnetosphere, Philos. Trans. R. Soc. London, Ser. A, 358 , 2471.

Connerney, J. E. P., et al. (1993), Images of excited $\mathrm{H}_{3}^{+}$at the foot of the Io flux tube in Jupiter's atmosphere, Science, 262, 1035.

Connerney, J. E. P., M. H. Acuña, N. F. Ness, and T. Satoh (1998), New models of Jupiter's magnetic field constrained by the Io flux tube footprint, J. Geophys. Res., 103, 11,929.

Crary, F. J. (1997), On the generation of an electron beam by Io, J. Geophys. Res., 102, 37.

Crary, F. J., and F. Bagenal (1997), Coupling the plasma interaction at Io to Jupiter, Geophys. Res. Lett., 24, 2135.

Delamere, P. A., F. Bagenal, R. E. Ergun, and Y.-J. Su (2003), Momentum transfer between the Io plasma wake and Jupiter's ionosphere, J. Geophys. Res., 108(A6), 1241, doi:10.1029/2002JA009530.

Dols, V., J. C. Gérard, V. Clarke, J. Gustin, and D. Grodent (2000), Diagnostics of the Jovian aurora deduced from ultraviolet spectroscopy: Model and GHRS observations, Icarus, 147, 251.
Frank, L. A., and W. R. Paterson (1999), Intense electron beams observed at Io with the Galileo spacecraft, J. Geophys. Res., 104, 28,657.

Frank, L. A., et al. (1996), Plasma observations at Io with the Galileo spacecraft, Science, 274, 394.

Gérard, J. C., and V. Singh (1982), A model of energetic electrons and EUV emission in the Jovian and Saturnian atmospheres and implications, J. Geophys. Res., 87, 4525

Gérard, J.-C., J. Gustin, D. Grodent, P. Delamere, and J. T. Clarke (2002), Excitation of the FUV Io tail on Jupiter: Characterization of the electron precipitation, J. Geophys. Res., 107(A11), 1394, doi:10.1029/ 2002JA009410

Goertz, C. K. (1980), Io's interaction with the plasma torus, J. Geophys. Res., 85, 2949.

Goldreich, P., and D. Lynden-Bell (1969), Io: A Jovian unipolar inductor, Astrophys. J., 156, 59.

Grodent, D., J. H. Waite Jr., and J. C. Gérard (2001), A self-consistent model of the Jovian auroral thermal structure, J. Geophys. Res., 106, 12,933

Grodent, D., J. T. Clarke, J. Kim, J. H. Waite Jr., and S. W. H. Cowley (2003), Jupiter's main auroral oval observed with HST-STIS, J. Geophys. Res., 108(A11), 1389, doi:10.1029/2003JA009921.

Gurnett, D. A., and C. K. Goertz (1981), Multiple Alfvén wave reflections excited by Io: Origin of the Jovian decametric arcs, J. Geophys. Res., 86, 717.

Hill, T. W., and V. M. Vasyliūnas (2002), Jovian auroral signature of Io's corotational wave, J. Geophys. Res., 107(A12), 1464, doi:10.1029/ 2002JA009514.

Hinson, D. P. A., J. Kliore, F. M. Flasar, J. D. Twicken, P. J. Schinder, and R. G. Herrera (1998), Galileo radio occultation measurements of Io's ionosphere and plasma wake, J. Geophys. Res., 103, 29,343.

Kivelson, M. G., F. Bagenal, W. S. Kurth, F. M. Neubauer, C. Paranicas, and J. Saur (2004), Magnetospheric interactions with satellites, in Jupiter: The Planet, Satellites, and Magnetosphere, edited by F. Bagenal, T. E. Dowling, and W. B. McKinnon, p. 513, Cambridge Univ. Press, New York

Leblanc, Y. (1981), On the arc structure of the DAM Jupiter emission, J. Geophys. Res., 86, 8546.

Leblanc, Y., G. A. Dulk, and F. Bagenal (1994), On Io's excitation and the origin of Jupiter's decametric radiation, Astron. Astrophys., 290, 690.

Neubauer, F. M. (1980), Nonlinear standing Alfvén wave current system at Io: Theory, J. Geophys. Res., 85, 1171.

Prangé, R., D. Rego, L. Pallier, J. E. R. Connerney, P. Zarka, and J. Queinnec (1998), Detailed study of FUV Jovian auroral features with the postCOSTAR HST faint object camera, J. Geophys. Res., 103, 20,195.

Queinnec, J., and P. Zarka (1998), Io-controlled decameter arcs and IoJupiter interaction, J. Geophys. Res., 103, 26,649.

Russell, C., and D. Huddleston (2000), The unipolar inductor myth: Mass addition or motional electric field as the source of field-aligned currents at Io, Adv. Space Res., 26, 1665.

Saur, J. (2004), A model of Io's local electric field for a combined Alfvénic and unipolar inductor far-field coupling, J. Geophys. Res., 109, A01210, doi:10.1029/2002JA009354.

Saur, J., F. M. Neubauer, D. F. Strobel, and M. E. Summers (1999), Threedimensional plasma simulation of Io's interaction with the Io plasma torus: Asymmetric plasma flow, J. Geophys. Res., 104, 25,105.

Saur, J., F. M. Neubauer, J. E. P. Connerney, P. Zarka, and M. G. Kivelson (2004), Plasma interaction of Io with its plasma torus, in Jupiter: The Planet, Satellites, and Magnetosphere, edited by F. Bagenal, T. E. Dowling, and W. B. McKinnon, p. 537, Cambridge Univ. Press, New York.

Vasavada, A. R., et al. (1999), Jupiter's visible aurora and Io footprint, J. Geophys. Res., 104, 27,133.

Waite, J. H., Jr., T. E. Cravens, J. U. Kozyra, A. F. Nagy, S. K. Atreya, and R. H. Chen (1983), Electron precipitation and related aeronomy of the Jovian thermosphere and ionosphere, J. Geophys. Res., 88, 6143.

Williams, D. J., B. H. Mauk, R. E. McEntire, E. C. Roelof, T. P. Armstrong, B. Wilken, J. G. Roederer, S. M. Krimigis, T. A. Fritz, and L. J. Lanzerotti (1996), Electron beams and ion composition measured at Io and in its torus, Science, 274, 401

J. T. Clarke, Department of Astronomy, Center for Space Physics, 725 Commonwealth Avenue, Boston, MA 02215, USA.

J.-C. Gérard, D. Grodent, and A. Saglam, Institut d'Astrophysique et de Géophysique, Laboratoire de Physique, Atomosphérique et Planétaire, Université de Liège, Bat. B5c, Allee du 6 Aout, Liege B-4000, Belgium. (jc.gerard@ulg.ac.be) 